\title{
Effects of Animal Care Procedures on Plasma Corticosterone Levels in Group-Housed Mice during the Nocturnal Active Phase
}

\author{
Hiroyuki SAKAKIBARA ${ }^{1,2) *}$, Akiharu KOYANAGI ${ }^{1) *}$, Taka-aki SUZUKI ${ }^{3)}$, \\ Atsumi SUZUKI ${ }^{1)}$, Lin LING $^{1)}$, and Kayoko SHIMOI ${ }^{1,2,4)}$ \\ ${ }^{1)}$ Graduate School of Nutritional and Environmental Sciences, ${ }^{2)}$ Institute for Environmental Sciences, and \\ ${ }^{4)}$ Global COE program, University of Shizuoka, 52-1 Yada, Suruga-ku, Shizuoka 422-8526, and \\ ${ }^{3)}$ Shizuoka Industrial Research Institute, 2078 Makigaya, Aoi-ku, Shizuoka 421-1298, Japan
}

\begin{abstract}
C57BL/6 mice were housed five per cage on a 12:12 h light/dark cycle. All animal care, including bed cleaning, was carried out during the nonactive phase. After 2 weeks, mean plasma corticosterone levels, collected during the nonactive (ZT6) and active (ZT18) phases, were 66.0 and $270.9 \mathrm{ng} / \mathrm{ml}$, respectively. The values at ZT18 gradually increased in the order of the mice used for blood collection, but not at ZT6. When animal care was carried out at ZT18, the increasing pattern of plasma corticosterone levels previously observed at ZT18 was less pronounced after 2 weeks of acclimatization, and was not observed after 4 weeks. Therefore, animal care should be carried out in the active phase for at least 4 weeks before experiments involving stress responses in the active phase.
\end{abstract}

Key words: active phase, animal care, stress

Stress induction in rodents is a critical element in the study of the neural and neuroendocrine mechanisms involved in establishing and maintaining a state of stress. Stress can lead to physical and psychiatric pathologies in rodents, and knowledge of the biologic mechanisms may lead to the development of effective means for relieving significant human suffering and distress [11]. Recently, many research groups, including ours, have studied how to cure and/or treat stress-related illnesses using models of animal stress [3,11, 19-21,24]. Many of these studies employed some biological factors, socalled stress biomarkers, to evaluate physiological changes under stressful conditions. When exposed to stress, the hypothalamic-pituitary-adrenal (HPA) axis is activated and consequently glucocorticoids, namely corticosterone in rodents and cortisol in humans, are secreted into blood from the adrenal cortex [7,17]. Interestingly, this secretion into blood is recognized as yielding a common stress response in rodents to all kinds of stress, such as restraint, electrical foot-shock, cold, forced swimming, isolation, or confrontation, although secretion intensities vary among individual kind of stress $[3,14,19]$. Hence, researchers can evaluate stress-relative responses in rodents under stressful conditions by measuring blood corticosterone, and can research and screen anti-stress dietary ingredients and drugs. On the other hand, almost all manual work for experiments such as dissection, blood collection, and measurement of body

(Received 14 April 2010 / Accepted 28 June 2010)

Address corresponding: K. Shimoi, Institute for Environmental Sciences, University of Shizuoka, 52-1 Yada, Suruga-ku, Shizuoka 422-8526, Japan

*These authors contributed equally to this work. 
(A)

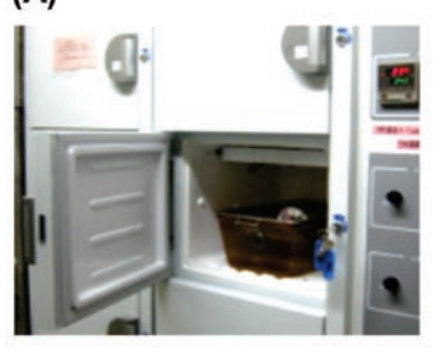

(B)

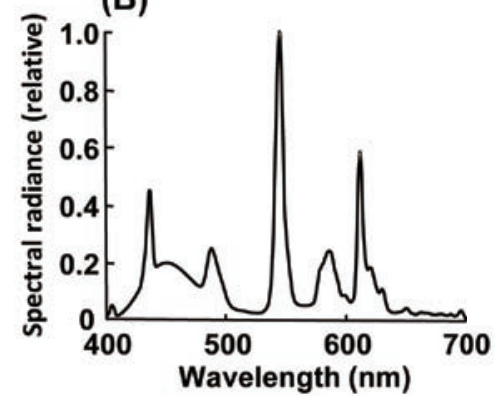

(C)

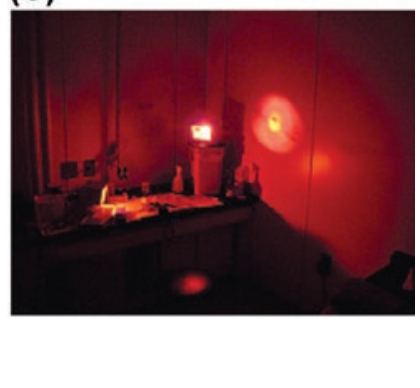

Fig. 1. Environments of the housing chamber and dissecting room used in this study. (A) Individual animal breeding system; (B) spectral radiance distribution of the light source, a cold cathode fluorescent lamp (4.2 W), used in the chamber. Its intensity was set at $250 \mathrm{~lx}$ during the light period. The light cycle was a 12-h dark/12-h light cycle; (C) dissecting room with red light as illumination.

weight and food intake is usually performed while the animal room is illuminated, even though rodents are nocturnal. However, Dubovicky et al. reported that shaker stress increased plasma corticosterone levels with a greater absolute value during the dark period (active phase) than the light period (nonactive phase) [8]. Bernatova et al. reported that mean arterial pressure was greater in the nonactive phase under shaker stress than in the active phase, and consequently that stress delivered during the nonactive phase represents a higher cardiovascular risk [4]. This background indicates that physiological responses to stress exposure are quite different between the active and nonactive phases in rodents, even when exposed to the same stress, and that stress experiments using nocturnal animals should be held not only during the active phase but also during the nonactive phase. Accordingly, in animal experiments, physiological stress responses to environmental stressors, except purposeful factors, should be reduced as much as possible to minimize their effects on results. However, data about physiological stress responses during the active phase, especially for mice housed in groups, is limited. In this study, we investigated acclimatization procedures for stress experiments in the active phase on group-housed mice and report that stresslike physiological responses at dissection can be avoided.

Male C57BL/6NCrSlc mice (5 weeks old) were purchased from Japan SLC (Shizuoka, Japan), and were immediately housed five mice per cage $(338 \times 225 \times 140$ $\mathrm{mm})$. Mice had access to a certified diet for mice (MF) from the Oriental Yeast Co. (Tokyo, Japan) and tap water. Each cage was individually kept in the Individual Animal Breeding System for Mice (LP-30CCFL-8ARS, Nippon Medical \& Chemical Instruments, Osaka, Japan) as shown in Fig. 1A. The insides of individual chambers were maintained at $23 \pm 1{ }^{\circ} \mathrm{C}$ under 12 -h dark/12-h light cycles. A cold cathode fluorescent lamp (4.2 W) was used as the illumination source in the chamber, and its intensity was adjusted to illuminate the floor of the chamber by $250 \mathrm{~lx}$. Its spectral radiance was measured using a spectral radiometer CS-1000A (Konica Minolta Holdings, Inc., Tokyo, Japan) and is shown in Fig. 1B. All experimental procedures were in accordance with the guideline of the University of Shizuoka, Japan, for the Care and Use of the Laboratory Animals, based on those of the American Association for Laboratory Animal Science.

Protocol-1: All daily care was performed at ZT6. "ZT" is an abbreviation of Zeitgeber time, and "ZTO" indicated the period when the light went on. Hence, the light period was from ZT0 to ZT12, and the dark period was from ZT12 to ZT24 (0). Our daily care included changing the bedding, refreshing the food and water bottles twice a week, and measurements of body weight once a week. We experimented on mice at ZT6 and ZT18 as described below, after acclimatization for 2 weeks under the well-regulated environment. Environmental factors, such as noise and light, have been reported to induce stress responses $[9,12,26,27]$. In this study, mice were individually taken from the Individual Animal Breeding System (Fig. 1A), put into a desiccator covered 
with an aluminum foil, and then immediately transferred to the dissecting room (Fig. 1C). Therefore, the other mice could not have known what was happening to their neighbors, including either though smell or noise, except that a mouse housed in their cage had been taken out. Mice are largely insensitive to red light $(>650 \mathrm{~nm})[5$, 13]. Hence, red light was used for illumination in the dissecting room, and its luminosity was reduced to around $20 \mathrm{~lx}$ in order to diminish the physiological effect induced by light exposure. Furthermore, a flashlight covered with red film was used as the light source, when removing mice during the dark period. Mice were anesthetized with ether, and blood was collected from the abdominal vein using heparinized tubes (Capiject, Terumo Medical Corporation, Somerset, NJ, USA) within 3 min of their removal from the cage. The five mice in each cage were taken out at 8 min intervals. Plasma was separated by centrifugation at $1,200 \mathrm{~g}$ for $10 \mathrm{~min}$ and maintained at a temperature of $-80^{\circ} \mathrm{C}$ until corticosterone analysis.

Protocol-2: All daily care was performed at ZT18 under the red light with reduced luminosity. After 2 weeks of acclimatization, mice were anesthetized with ether at ZT18, and blood was collected.

Protocol-3: All daily care was performed at ZT18 under the red light with reduced luminosity. After 4 weeks of acclimatization, mice were anesthetized with ether at ZT18, and blood was collected.

Plasma corticosterone levels were determined in duplicate using an EIA kit (Assay Designs, Inc., Ann Arbor, MI, USA) according to the manufacturer's protocol. Statistical analyses were performed using Student's $t$-test with Pharmaco Analyst II (Hakuhousha Co., Ltd., Tokyo, Japan). A $P$ value of less than 0.05 was considered to indicate a statistically significant difference.

Plasma corticosterone levels were analyzed individually at ZT6 and ZT18 after 2 weeks of acclimatization including daily animal care at ZT6. As shown in Fig. 2, the mean-value in the active phase $(270.9 \mathrm{ng} / \mathrm{ml})$ was significantly higher than that of the nonactive phase $(66.0$ $\mathrm{ng} / \mathrm{ml}$ ). Corticosterone is secreted into blood from the adrenal cortex, when the HPA axis is activated, for example, under stressful conditions as described above. Additionally, the activity of the HPA axis is recognized to have a diurnal pattern, and therefore glucocorticoid

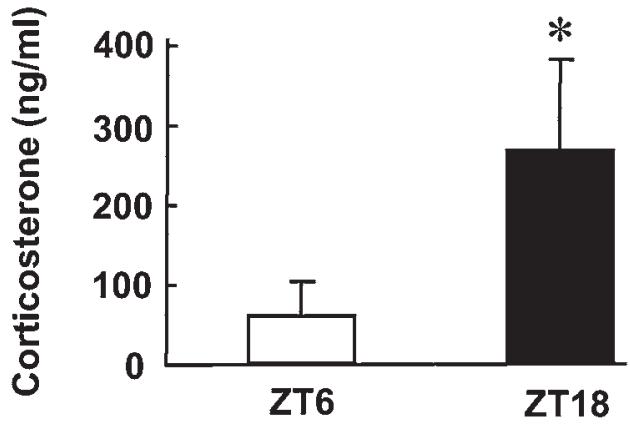

Fig. 2. Plasma corticosterone levels during the nonactive phase (ZT6) and active phase (ZT18) in mice. After 2 weeks of animal care procedure performed at ZT6, blood was collected individually at ZT6 and ZT18. Values are presented as mean $\pm \mathrm{SD}(\mathrm{n}=5) . * P<0.05$ vs ZT6 (Student's $t$-test).

production indicates a robust diurnal pattern [23, 28]. Briefly, the blood corticosterone level is lowest at ZT04 , and then starts to increase. It reaches a peak at ZT1216 under 12-h dark/12-h light cycles [8, 18, 25, 28], indicating that plasma corticosterone levels are higher in the first half of the active phase than the first half of the nonactive phase. The same pattern is seen for cortisol in humans [23]. Our results are in agreement with the reported, normal physiological responses; however, the amplitude of plasma corticosterone levels between the active and nonactive phases in our results was about two-times larger than that those reported by other researchers $[18,25,28]$. Interestingly, our values for corticosterone in the active phase gradually increased in the order of the mice used for blood collection: first mouse of five, $83.3 \mathrm{ng} / \mathrm{ml}$, then $317.2,308.6,273.0$, and $372.6 \mathrm{ng} / \mathrm{ml}$; the values in the nonactive phase were 17.3 $\mathrm{ng} / \mathrm{ml}$, then 115.2, 83.0, 32.3, and $82.5 \mathrm{ng} / \mathrm{ml}$ (Fig. 3A and $3 \mathrm{~B}$ ), respectively. Blood collection is a very common procedure performed on laboratory animals and is well-known to induce stress [1,22]. Croft and co-workers reported that a stress response was evident five minutes after saline injections to the abdomen [6]. Therefore, we employed ether aspiration as anesthesia to minimize stress responses during the handling and injection of mice. Although exposure to ether has also been reported to increase the plasma corticosterone level [15], we considered that some stimuli, for example, contact 
(A)

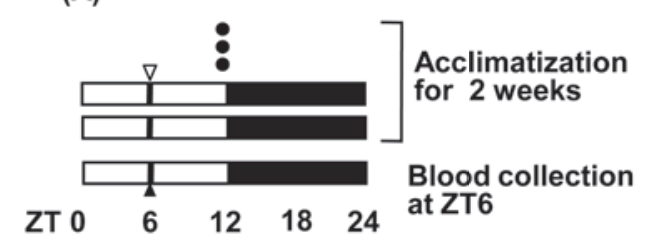

(B)

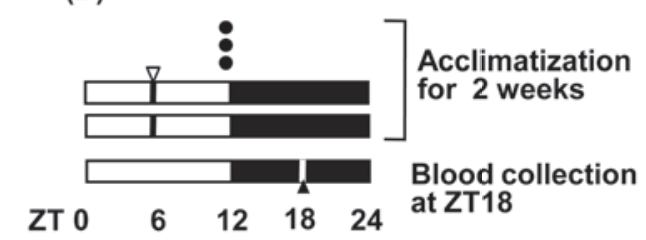

(C)

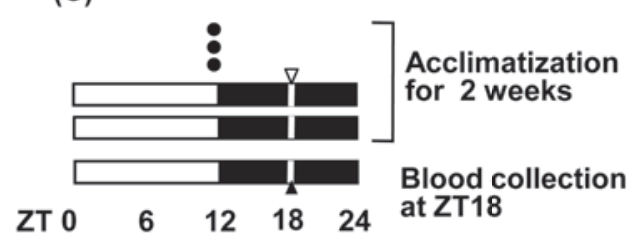

(D)

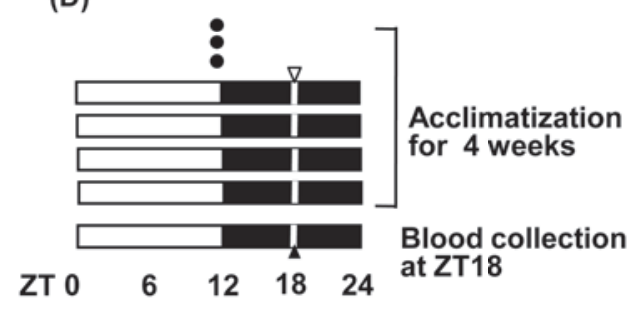

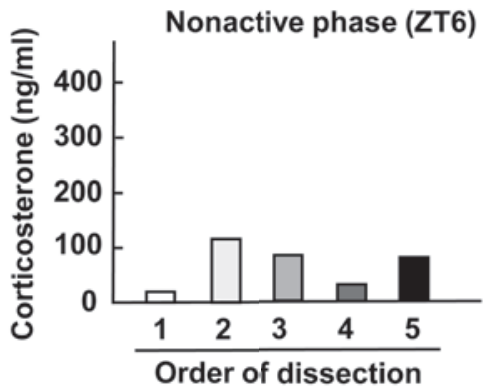
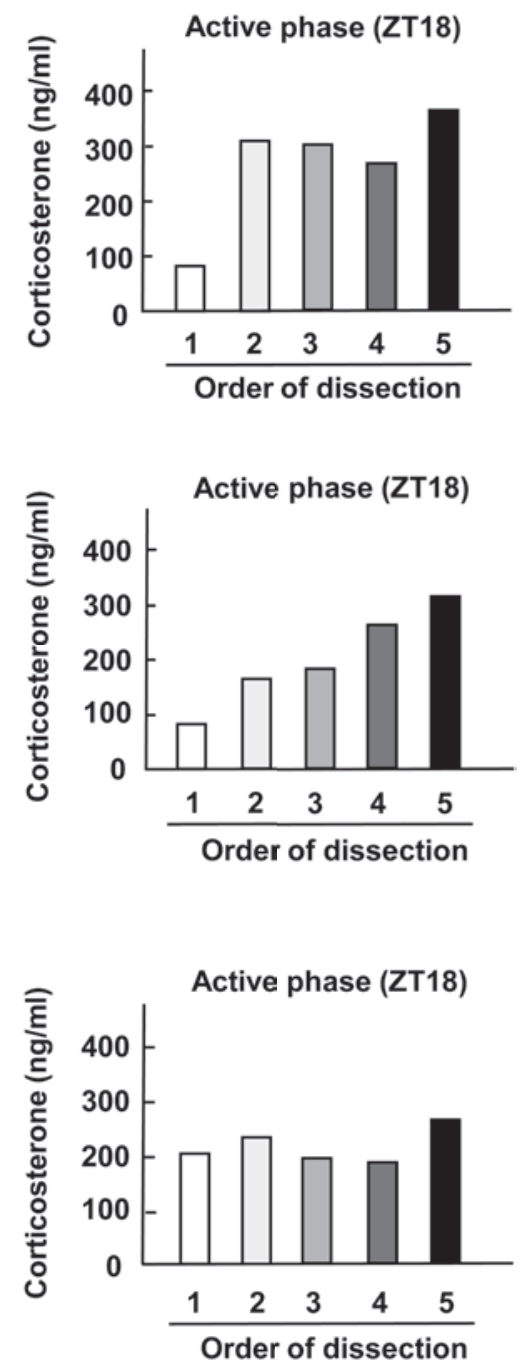

Fig. 3. Plasma corticosterone of individual mice collected during the nonactive phase (ZT6) and active phase (ZT18). After 2 weeks of animal care procedure performed at ZT6, blood was collected individually at ZT6 (A) and ZT18 (B). After 2 weeks of animal care procedures performed at ZT18, blood was collected at ZT18 (C). After 4 weeks of animal care procedure performed at ZT18, blood was collected at ZT18 (D). $\nabla$, period of daily animal care; $\boldsymbol{\Delta}$, period of blood collection. Plasma samples were collected from each mouse ( 5 per cage) at 8 min intervals. 
with humans, affected the mice except for the first mouse during the active phase. Therefore, the stimulation of corticosterone might not have appeared during the active phase in mice housed singly in previous experiments $[8$, 18, 25]. To accustom the mice to human contact, we next tried analyzing plasma corticosterone levels after carrying out daily animal care during the active phase, ZT18. After 2 weeks of acclimatization, blood was collected at ZT18 and the corticosterone levels were analyzed. The values for the first and fifth mice were 81.8 and $315.1 \mathrm{ng} / \mathrm{ml}$, respectively (Fig. 3C), indicating that the values were similar to those obtained after carrying out daily animal care during the nonactive phase (Fig. 3B). However, the corticosterone levels of the second, third and fourth mice showed a gradual increase, and this change was not as dramatic as initially observed. These results indicate that daily animal care performed during the active phase for two weeks diminished the stress response of the group-housed mice during the active phase. When rodents are exposed to stressors such as injection, their corticosterone levels increase in order of exposure [6], indicating that the second and subsequent mice still suffered some stress in the Protcol-2 (Fig. 3C). In addition, the data shown in Fig. 3B and 3C indicate that corticosterone level in the initial mouse of five might be intact level without stress responses. When the acclimatization continued for 4 weeks, plasma corticosterone was $216.8 \pm 32.5 \mathrm{ng} / \mathrm{ml}$, and did not increase in accordance with the order of the mice for blood collection (Fig. 3D). The plasma corticosterone the value of the first mouse $(205.6 \mathrm{ng} / \mathrm{ml})$ was higher than in the other protocols (Fig. 3B and 3C). The reason for this difference might be that the animals were older, since the acclimatization period was longer in this experiment, and blood corticosterone levels are known to increase with age $[2,10,16]$. Especially, Lin et al. reported that serum corticosterone levels in young mice gradually increased from 3 weeks of age to 14 weeks [16].

The results of our study indicate that daily animal care performed during animal experiments may affect the stress response in the active phase. Group-housed mice, for example five mice housed per cage, showed a typical stress response for the blood collection in the active phase, when daily animal care procedures were carried out during the nonactive phase. To avoid misunderstand- ing the stress-related responses, daily animal care should be done during the active phase for at least 4 weeks before the experiment is conducted during the active phase using group-housed mice. We believe that our results will be helpful for researchers investigating physiological responses to stress in rodents during the active phase and novel anti-stress foods and drugs.

\section{Acknowledgments}

This work was supported in part by a Grant-in-aid for Young Scientists (B) from the Japan Society for the Promotion of Science (21700761), the Global COE Program, the Center of Excellence for Innovation in Human Health Sciences, and from the Ministry of Education, Culture, Sports, Science and Technology of Japan.

\section{References}

1. Abatan, O.I., Welch, K.B., and Nemzek, J.A. 2008. J. Am. Assoc. Lab. Anim. Sci. 47: 8-15.

2. Akana, S.F. 2008. Physiol. Behav. 94: 569-579.

3. Andersen, M.L., Bignotto, M., Machado, R.B., and Tufik, S. 2004. Braz. J. Med. Biol. Res. 37: 791-797.

4. Bernatova, I., Key, M.P., Lucot, J.B., and Morris, M. 2002. Hypertension 40: 768-773.

5. Conway, B.R. 2007. Curr. Biol. 17: R457-460.

6. Croft, A.P., O'Callaghan, M.J., Shaw, S.G., Connolly, G., Jacquot, C., and Little, H.J. 2008. Brain Res. 1238: 1222.

7. Diorio, D., Viau, V., and Meaney, M.J. 1993. J. Neurosci. 13: 3839-3847.

8. Dubovicky, M., Mach, M., Key, M., Morris, M., Paton, S., and Lucot, J.B. 2007. Neuro. Endocrinol. Lett. 28: 846853.

9. Fonken, L.K., Finy, M.S., Walton, J.C., Weil, Z.M., Workman, J.L., Ross, J., and Nelson, R.J. 2009. Behav. Brain Res. 205: 349-354.

10. Harris, S.B., Gunion, M.W., Rosenthal, M.J., and Walford, R.L. 1994. Mech. Ageing Dev. 73: 209-221.

11. Heinrichs, S.C. and Koob, G.F. 2006. Curr. Protoc. Neurosci. Chapter 8: Unit8 4.

12. Ishida, A., Mutoh, T., Ueyama, T., Bando, H., Masubuchi, S., Nakahara, D., Tsujimoto, G., and Okamura, H. 2005. Cell Metab. 2: 297-307.

13. Jacobs, G.H., Williams, G.A., Cahill, H., and Nathans, J. 2007. Science 315: 1723-1725.

14. Kawabata, K., Kawai, Y., and Terao, J. 2009. J. Nutr. Biochem. (Epub ahead of print.)

15. Korosi, A., Schotanus, S., Olivier, B., Roubos, E.W., and Kozicz, T. 2005. Brain Res. 1046: 172-179.

16. Lim, T.S., Putt, N., Safranski, D., Chung, C., and Watson, R.R. 1981. Immunology 44: 289-295. 
17. Magarinos, A.M., Somoza, G., and De Nicola, A.F. 1987. Horm. Metab. Res. 19: 105-109.

18. Malisch, J.L., Breuner, C.W., Gomes, F.R., Chappell, M.A., and Garland, T. Jr. 2008. Gen. Comp. Endocrinol. 156: 210-217.

19. Miyashita, T., Yamaguchi, T., Motoyama, K., Unno, K.Y.N., and Shimoi, K. 2006. Biochem. Biophys. Res. Commun.349: 775-780.

20. Motoyama, K., Nakai, Y., Miyashita, T., Fukui, Y., Morita, M., Sanmiya, K., Sakakibara, H., Matsumoto, I., Abe, K., Yakabe, T., Yajima, N., and Shimoi, K. 2009. Physiol Genomics 37: 79-87.

21. Nishio, Y., Nakano, Y., Deguchi, Y., Terato, H., Ide, H., Ito, C., Ishida, H., Takagi, K., Tsuboi, H., Kinae, N., and Shimoi, K. 2007. Genes Environ. 29: 17-22.

22. O’Neill, P.J. and Kaufman, L.N. 1990. Lab. Anim. Sci. 40:
641-643.

23. Perry, M.G., Kirwan, J.R., Jessop, D.S., and Hunt, L.P. 2009. Ann Rheum Dis 68: 63-68.

24. Porsolt, R.D., Le Pichon, M., and Jalfre, M. 1977. Nature 266: 730-732.

25. Richard, E.M., Helbling, J.C., Tridon, C., Desmedt, A., Minni, A.M., Cador, M., Pourtau, L., Konsman, J.P., Mormede, P., and Moisan, M.P. Endocrinology 151: 649659.

26. Turner, J.G., Parrish, J.L., Hughes, L.F., Toth, L.A., and Caspary, D.M. 2005. Comp. Med. 55: 12-23.

27. Willott, J.F. 2007. J. Am. Assoc. Lab. Anim. Sci. 46: $23-$ 27.

28. Yang, S., Liu, A., Weidenhammer, A., Cooksey, R.C., McClain, D., Kim, M.K., Aguilera, G., Abel, E.D., and Chung, J.H. 2009. Endocrinology 150: 2153-2160. 